\title{
Isolation of recombinant fragments of the major outer-membrane protein of Chlamydia trachomatis: their potential as subunit vaccines
}

\author{
J. W. Conlan, ${ }^{*}$ S. Ferris, I. N. Clarke and M. E. Ward \\ Department of Microbiology, University of Southampton Medical School, Southampton General Hospital, \\ Tremona Road, Southampton SO9 $4 X Y$, UK
}

(Received 15 March 1990; revised 21 May 1990; accepted 13 June 1990)

\begin{abstract}
Recombinant fragments of the major outer-membrane protein (MOMP) of Chlamydia trachomatis, expressed at high levels in Escherichia coli, were isolated and purified. Antisera to the recombinant proteins reacted preferentially with overlapping synthetic peptides covering the immunoaccessible variable segments of MOMP. These sera also reacted in a species-specific manner with the surface of intact infectious elementary bodies, and in a Chlamydia genus-specific manner in assays using denatured or bound chlamydial antigens. The ability of recombinant MOMP preparations to elicit antibody to the surface of chlamydial elementary bodies raises the possibility that these proteins may be useful for chlamydial vaccine development.
\end{abstract}

\section{Introduction}

Chlamydiae are obligate intracellular bacterial pathogens. In developing countries Chlamydia trachomatis serovars $A, B$ and $C$ are a major infectious cause of preventable blindness (trachoma). Worldwide, serovars $\mathrm{D}$ to $\mathrm{K}$ are an important cause of sexually transmitted disease and associated infertility (Robertson \& Ward, 1988). Early trachoma vaccine trials using whole chlamydiae were partially successful in that short-term serovar-specific protection was achieved, but disease of enhanced severity was reported in some individuals reinfected with heterologous serovars (reviewed in Schachter \& Dawson, 1978). In the ensuing search for a subunit vaccine which excludes these complications much attention has been focused on the major outermembrane protein (MOMP) of C. trachomatis.

MOMP represents up to $60 \%$ of the surface protein of the infectious elementary body (EB) and the intracellularly replicative reticulate body (RB; Caldwell et al., 1981). MOMP carries serovar-, subspecies-, species and genus-specific epitopes (Terho et al., 1982; Batteiger et al., 1986; Conlan et al., 1988). Antibodies reactive with these epitopes form the basis for the serological classification of $C$. trachomatis (Wang \& Grayston, 1982). The location of these epitopes has been deter-

\footnotetext{
Abbreviations: EB, elementary body; mAb, monoclonal antibody; (r)MOMP, (recombinant) major outer-membrane protein; RB, reticulate body; TEM, transmission electron microscopy.
}

mined using monoclonal antibodies (mAbs) in conjunction with expressed recombinant DNA (Baehr et al., 1988) or synthetic peptide (Conlan et al., 1988; Stephens et al., 1988) probes. The gene and inferred amino acid sequences of MOMP for all $C$. trachomatis serovars have been either wholly or partially determined (Pickett et al., 1987; Stephens et al., 1987; Baehr et al., 1988; Yuan et al., 1989). The MOMP sequence for $C$. psittaci ewe abortion agent has also been determined (Pickett $e t$ al., 1988a). Comparisons of these sequences has provided a molecular rationale for the serological diversity of chlamydiae.

mAbs (Lucero \& Kuo, 1985; Zhang et al., 1987) to the surface-exposed serovar- and subspecies-specific epitopes of MOMP neutralize chlamydial infection. Species-specific antibody to MOMP generally fails to neutralize chlamydiae (Zhang et al., 1987) because the corresponding epitope is less well exposed (Kuo \& Chi, 1987). However, Collett et al. (1989) recently described a species-specific mAb which reacted with a surfaceexposed epitope of MOMP. This raises the possibility that a single component vaccine based on MOMP might be effective against all serovars of $C$. trachomatis.

Using recombinant DNA techniques, Pickett $e t$ al. $(1988 b)$ achieved high-level expression of recombinant serovar L1 MOMP (rMOMP) fragments in Escherichia coli. The present paper describes: (i) the facile purification of these recombinant protein fragments; (ii) the ability of these antigens to generate antibodies which 
react at the surface of viable chlamydiae in a broad species-specific manner.

\section{Methods}

Organisms. The production of stable recombinants of $E$. coli strain K12-JM109 expressing approximately $\frac{3}{4}, \frac{1}{2}$ or $\frac{1}{4}$-length fragments of C. trachomatis serovar L1 MOMP as insoluble cytoplasmic inclusions has been described previously (Pickett $e t$ al., 1988b). C. trachomatis serovar A (strain SA1), serovar B (strain B/Jali20/OT), serovar C (strain UW1), serovar D (strain 1883), serovar F (a recent clinical isolate), serovar G (strain IOL 238), serovar L1 (strain L1/440/LN) and C. psittaci (strain EAE A22/M) were grown and purified as previously described (Salari \& Ward, 1981).

Isolation of $r M O M P$. Stocks of recombinant bacteria were stored in liquid nitrogen. Thawed stock cultures were revived on Luria agar $(16 \mathrm{~h}$ at $\left.37^{\circ} \mathrm{C}\right)$ containing ampicillin $\left(50 \mathrm{mg}^{-1}\right)$ and IPTG $(0.1 \mathrm{~mm})$ as inducer. These cultures were used to inoculate $100 \mathrm{ml}$ volumes of Luria broth (as above but with the agar omitted) which were incubated aerobically at $37^{\circ} \mathrm{C}$ for $16 \mathrm{~h}$ in $500 \mathrm{ml}$ conical flasks on an orbital shaker. Cells were harvested by centrifugation and treated with lysozyme-EDTA (Osborn \& Munson, 1974). The resulting spheroplasts were lysed by sonication (MSE Soniprep, $3 \times 15 \mathrm{~s}$ at maximum amplitude) and the insoluble rMOMP inclusions were harvested by centrifugation (12000 r.p.m. for $5 \mathrm{~min}$, microcentrifuge) and washed twice in $20 \mathrm{~mm}$-Tris/HCl, $\mathrm{pH} 7.0$. Few intact cells remained as determined by interference microscopy. The washed pellet was treated with RNAase and DNAase for $1 \mathrm{~h}$ at $37^{\circ} \mathrm{C}$ in the presence of $\mathrm{MgCl}_{2}$ (50 mM), washed again, then solubilized at $50^{\circ} \mathrm{C}$ for $30 \mathrm{~min}$ in $20 \mathrm{mM}$ Tris/ $\mathrm{HCl}$, $\mathrm{pH} 7.0$ containing $5 \%(\mathrm{w} / \mathrm{v})$ SDS. $\beta$-Mercaptoethanol was added to $5 \%(\mathrm{v} / \mathrm{v})$, the mixture heated to $100^{\circ} \mathrm{C}$ for $5 \mathrm{~min}$, cooled and applied to a Sephadex $G 50$ column $(1.8 \times 28 \mathrm{~cm})$. The column was eluted with $50 \mathrm{mM}$-Tris/ $\mathrm{HCl}, \mathrm{pH} 7 \cdot 0$, containing $10 \mathrm{mM}$-EDTA, $1 \mathrm{mM}$ phenylmethylsulphonyl fluoride (PMSF), $0.1 \%$ (w/v) SDS and $0.05 \%$. (w/v) sodium azide at a flow rate of $22 \mathrm{ml} \mathrm{h}^{-1}$ and $5 \mathrm{ml}$ fractions were collected. Fractions were analysed individually by dot immunoblot using a mAb specific for $C$. trachomatis MOMP. Protein content of the fractions was determined with the Bradford reagent (Bio-Rad). The yield of rMOMP was $10-30 \mathrm{mg}$ (protein) per litre of culture.

Antisera. For antibody production pooled fractions from the Sephadex column were dialysed against distilled water, the resulting pellet resuspended in PBS and emulsified with incomplete Freund's adjuvant (IFA). Alternatively, the pooled fractions were adsorbed directly to alhydrogel without prior dialysis and washed twice in phosphate-buffered saline (Dulbecco A). Half-lop rabbits were prebled then immunized at two intramuscular and two subcutaneous sites with $200 \mu \mathrm{g}$ protein in either Freund's adjuvant or alhydrogel on days 1 , $28,63,114$ and serum obtained on day 140 . Rabbit antiserum against $E$. coli strain JM109 was kindly provided by Dr John Heckels of this department. This was produced by immunization with $100 \mu \mathrm{g}$ (as protein) antigen at multiple subcutaneous sites on days $1,14,28,45$ and 80; primary immunization was in complete Freund's adjuvant and all booster injections were in IFA. Antiserum was obtained on day 92. $\mathrm{mAbs} 6 \mathrm{E}$ and $2 \cdot 3$ were produced in $\mathrm{Balb} / \mathrm{c}$ mice by standard techniques (Terho et al., 1982) using purified C. trachomatis serovar L1 organisms as antigen.

ELISA for antibody. Antisera were titrated by an indirect ELISA in 96-well PVC microtitre trays (high activated, Flow Laboratories) using a published method (Robertson et al., 1987). For the detection of antibodies against chlamydiae and $E$. coli, the wells were coated overnight at room temperature with whole organisms $(5 \mu \mathrm{g}$ protein $\mathrm{ml}^{-1}, 100 \mu \mathrm{l}$ per well) in $0.05 \mathrm{M}$-carbonate buffer; $\mathrm{pH} 9.6$, containing
$0.01 \%(\mathrm{w} / \mathrm{v})$ sodium azide. For the detection of antibodies to rMOMP, washed inclusions were solubilized in $8 \mathrm{M}$-urea and diluted to $1 \mu \mathrm{g} \mathrm{ml}^{-1}$ in coating buffer immediately prior to use. The test sera were titrated through a threefold dilution series in assays with rMOMP fragments or $C$. trachomatis and through a twofold series in assays with E. coli. Endpoint titres were defined as the last well to give a positive reaction compared to controls.

Immunoblotting. Immunoblotting of chlamydia was done as previously described (Conlan et al., 1989b). Primary rabbit antisera were used at 1:200 dilution; $m A$ bs were used at 1:1000 dilution and were detected with goat anti-mouse IgG $(\mathrm{H}+\mathrm{L})$, alkaline phosphatase conjugate (Bio-Rad, 1:3000).

Immunogold-labelling. Surface-exposed epitopes of MOMP on intact chlamydiae were detected by transmission electron microscopy (TEM) using immunogold as previously described (Conlan et al., 1989 b). Test antisera were used at dilutions of $1: 10$ and 1:50; the protein $G$-gold conjugate was used at 1:10.

Epitope mapping. Solid-phase peptides for epitope mapping were synthesized according to a published method (Geysen et al., 1984) using a commercial kit (Cambridge Research Biochemicals). Synthesis was done according to manufacturer's instructions and as previously described (Conlan et al., 1988). A series of duplicate decapeptides spanning the entire serovar L1 MOMP sequence (Pickett et al., 1987) was synthesized. Each peptide overlapped the preceding decapeptide by five amino acids.

\section{Results}

\section{Analysis of rMOMP inclusions}

Cytoplasmically located, insoluble rMOMP inclusions were isolated, solubilized and analysed by SDS-PAGE (Fig. 1). Major proteins with apparent molecular masses of 13.5 and $32.7,28.2$ and $13.2 \mathrm{kDa}$ were obtained from the $E$. coli recombinants expressing respectively $\frac{3}{4}$, $\frac{1}{2}$ - or $\frac{1}{4}$-length fragments of serovar L1 MOMP (molecular mass approx. $40 \mathrm{kDa}$ ). All samples contained numerous faint minor bands, several of which reacted on Western blots with a mAb specific for C. trachomatis MOMP (Fig. 2). These were not removed by gel filtration on Sephadex G50. This $\mathrm{mAb}$ did not react with the $13.5 \mathrm{kDa}$ band of the $\frac{3}{4}$-rMOMP preparation nor with normal E. coli (not shown). The identity of this $13.5 \mathrm{kDa}$ band, which was not present in the other recombinant samples, is unknown.

\section{Production of antisera against rMOMP fragments}

Immunization with recombinant proteins in both alhydrogel and Freund's adjuvants produced high-titre antibodies reactive with all MOMP fragments on ELISA (Table 1). All of the antisera reacted in a genus-specific manner when whole chlamydiae were used as ELISA antigen. Pre-immune sera were negative for both assays. Antiserum raised against $E$. coli strain JM109 reacted with all rMOMP preparations, presumably due to the 


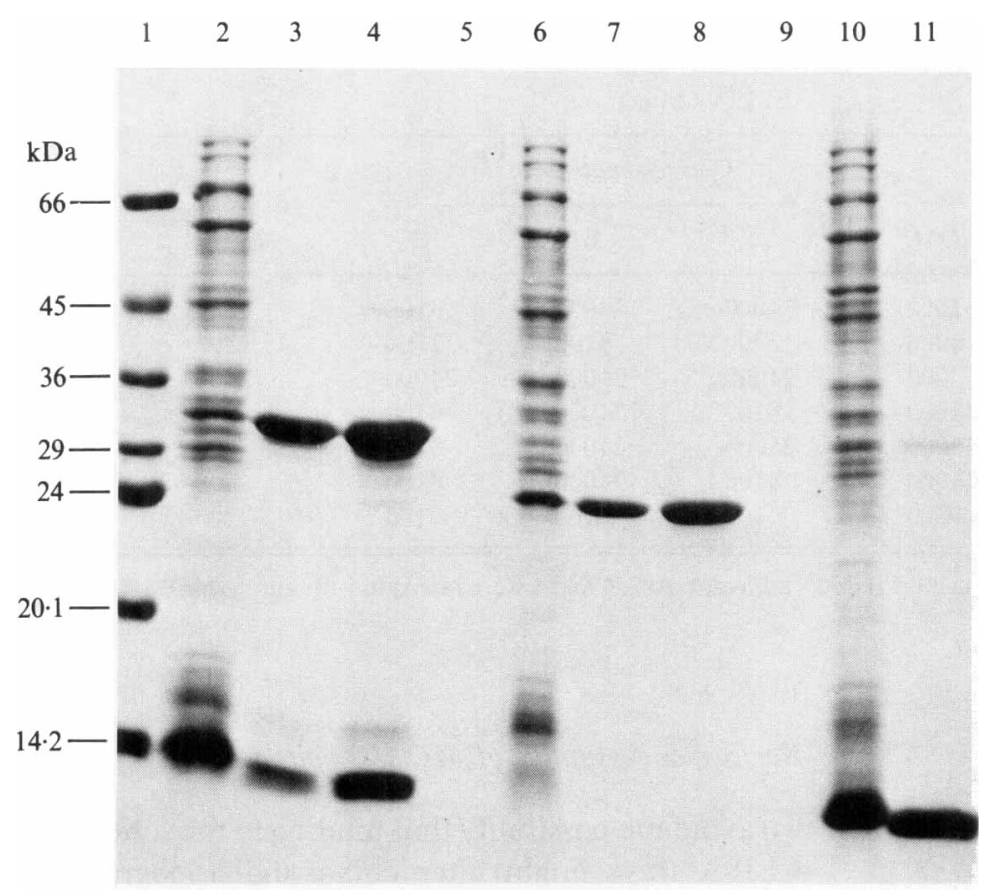

Fig. 1. SDS-PAGE analysis of rMOMP fragments. Lanes: 1 , molecular mass markers; $2, E$. coli expressing $\frac{3}{4}$-rMOMP; 3, isolated $\frac{3}{4}$ rMOMP inclusions; $4, \frac{3}{4}$ rMOMP from Sephadex G50; 5, blank; 6, E. coli expressing $\frac{1}{2}$-rMOMP; 7, $\frac{1}{2}$ rMOMP inclusions; $8, \frac{1}{2}$ rMOMP from Sephadex G50; 9, blank; 10, E. coli expressing $\frac{1}{4}$-rMOMP; $11, \frac{1}{4}$-rMOMP inclusions.

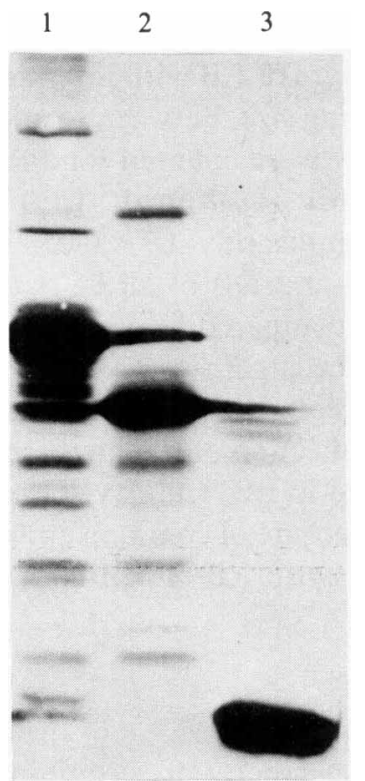

Fig. 2. Western blot of rMOMP fragments. Lanes: $1, \frac{3}{4}$-rMOMP purified inclusions; 2, t-rMOMP purified inclusions; 3, $\frac{1}{4}$-rMOMP purified inclusions. Lanes 1-3 probed with mAb reactive with a species-specific epitope on $C$. trachomatis MOMP.

presence of $E$. coli contaminants, since the same serum did not react with whole chlamydial antigens. Likewise rMOMP antisera reacted with whole $E$. coli antigens. This reactivity was abrogated by adsorption of the rMOMP antisera with whole $E$. coli cells. This procedure did not affect the titre of the rMOMP antisera with whole $C$. trachomatis antigens. Apparent variations in reactivity of these sera with different antigen preparations were noted. In particular, the lower reactivity of antisera 2 and 4 with rMOMP fragments was not matched by an equal decrease in reactivity to whole $C$. trachomatis antigens. Whether this is a reproducible phenomenon reflecting differences in antigen presentation or is a result of variability in the immune response of individual animals is unknown.

\section{Reactivity of antibodies to rMOMP with chlamydiae}

The reactivity of antisera to rMOMP with reduced and denatured antigens of chlamydiae on Western blots was exemplified by As1 (Fig. 3, lanes 1-4). This antiserum reacted with multiple bands from $C$. trachomatis serovars L1, B (B-complex), C (C-complex) but with far fewer bands for $C$. psittaci; the positions of the 'native' MOMP bands are indicated. One explanation for this might be the presence of cross-reactive epitopes on multiple chlamydial antigens. All antisera reacted in an identical manner. The most significant difference between the antisera was a marked decrease in the staining intensity of $C$. psittaci bands with antisera raised using alhydrogel as adjuvant. mAbs which were specific for MOMP reacted only with a single band on Western blot (Fig. 3, lane 5). The position of chlamydial LPS was determined using a Chlamydia genus-specific mAb (lane 6). All of the sera also reacted with $E$. coli strain JM109 on Western blots (Fig. 3, lanes 8 and 9). As1, 2 and 5 gave similar banding patterns with $E$. coli whilst the different blotting pattern of As6 was similar to that obtained with As3 and 
Table 1. Reactivity of antisera (ELISA titre) with rMOMP fragments and whole chlamydiae

\begin{tabular}{|c|c|c|c|c|c|c|c|c|c|}
\hline \multirow[b]{3}{*}{ Antiserum* } & \multicolumn{9}{|c|}{ ELISA antigen } \\
\hline & \multicolumn{4}{|c|}{ Recombinant fragments } & \multicolumn{3}{|c|}{ C. trachomatis serovars: } & \multirow{2}{*}{$\begin{array}{l}\text { C. psittaci } \\
\text { (EAE) }\end{array}$} & \multirow{2}{*}{$\begin{array}{r}\text { E. coli } \\
\text { (JM109) }\end{array}$} \\
\hline & & $\frac{3}{4}$-MOMP & $\frac{1}{2}$-MOMP & $\frac{1}{4}$ MOMP & $\mathbf{L l}$ & B & C & & \\
\hline Asl & & 437000 & 437000 & 437000 & 24000 & 24000 & 8100 & 8100 & 4000 \\
\hline As2 & & 16000 & 16000 & 16000 & 2700 & 8100 & 2700 & 300 & 12000 \\
\hline As3 & & 437000 & 437000 & 437000 & 24000 & 24000 & 24000 & 72000 & 4000 \\
\hline As4 & & 48000 & 48000 & 48000 & 8100 & 24000 & 8100 & 900 & 4000 \\
\hline As5 & . & 437000 & 437000 & 146000 & 24000 & 24000 & 8100 & 2700 & 4000 \\
\hline As6 & & 146000 & 146000 & 146000 & 8100 & 24000 & 8100 & 900 & 4000 \\
\hline E. coli antiserum & & 1800 & 5400 & 1800 & $<100$ & $<100$ & $<100$ & $<100$ & 36000 \\
\hline
\end{tabular}

*As1, 3 and 5 were raised to $\frac{3}{4}, \frac{1}{2}$-and $\frac{1}{4}$-rMOMP respectively using Freund's adjuvant. As2, 4 and 6 were raised to $\frac{3}{4}, \frac{1}{2}$ and $\frac{1}{4}$-rMOMP respectively using alhydrogel as adjuvant.

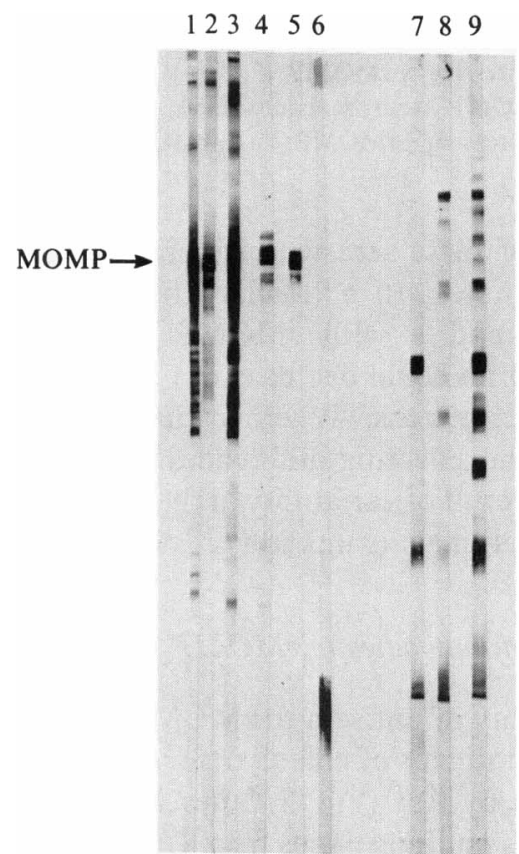

Fig. 3. Western blots of whole $C$. trachomatis with antisera against rMOMP. Antigens were: lanes 1-3, C. trachomatis serovars L1, B and C, respectively; lane 4, C. psittaci; lanes 5 and 6,C. trachomatis serovar C; lanes 7-9, E. coli strain JM109. Lanes 1-4 were probed with antisera to rMOMP; lane 5 with $C$. trachomatis MOMP-specific $\mathrm{mAb}$; lane 6 with chlamydia LPS-specific mAb; lane 7 with anti-JM109 antiserum; lanes 8 and 9 with antiserum to rMOMP.

4. When As1 and 4 were adsorbed with whole $E$. coli cells their reactivity to $E$. coli was abolished but the pattern of reactivity observed against chlamydiae was identical to blots using unadsorbed sera (not shown). Antiserum raised against $E$. coli did not react with chlamydiae on Western blots.

\section{Surface accessibility of MOMP epitopes}

To avoid the possibility that binding to the solid phase of ELISA trays might alter chlamydial topography, the surface accessibility of MOMP epitopes was determined by immunogold-labelling of intact chlamydiae in suspension prior to TEM. The purified chlamydial preparations used were a mixture of EB with intermediate bodies and some $R B$ of varying size. Between 15 and 30 organisms of each morphology were counted for each antiserum and chlamydial serovar examined. In general, the test sera reacted significantly $(P<0.05, t$-test of sample means) with $\mathrm{EB}$ and $\mathrm{RB}$ of all $C$. trachomatis serovars examined when compared to pre-immune sera or to the antiserum produced against $E$. coli (Table 2). In all cases RB showed a greater degree of labelling than did EB. This may be a reflection of the increased surface area of the RB compared to EB, and developmental differences in the surface exposure of common epitopes. None of the test sera reacted with $\mathrm{EB}$ or $\mathrm{RB}$ of $C$. psittaci.

\section{Epitope mapping}

The molecular specificity of recombinant antisera was examined against a series of solid-phase peptides spanning the entire serovar L1 MOMP sequence (Fig. 4). None of the test sera reacted with peptides corresponding to the $\mathrm{N}$-terminal- $\frac{1}{4}$ of L1 MOMP which is not present in the recombinant antigens (not shown). Antisera raised using Freund's adjuvant showed a wider spectrum of reactivity against peptides than did sera which employed alhydrogel as adjuvant. Pre-immune sera and antiserum to $E$. coli were negative in these assays. All antisera, with the exception of As5, reacted only with peptides which occur naturally in the respective immunizing MOMP fragments. We speculate that 
Table 2. Labelling of intact chlamydiae with rMOMP antisera by immunogold TEM

Figures represent the mean number of gold particles per organism.

\begin{tabular}{|c|c|c|c|c|c|c|c|c|c|}
\hline \multirow[b]{2}{*}{ Organism } & & \multicolumn{8}{|c|}{ Antiserum } \\
\hline & & Pre-bleed* & As1 & As2 & As 3 & As 4 & As5 & As6 & anti-JM109 \\
\hline \multirow[t]{2}{*}{ Serovar A } & EB & $0 \cdot 19$ & ND & ND & ND & ND & 11.9 & $8 \cdot 2$ & ND \\
\hline & RB & 0.69 & ND & ND & ND & ND & $43 \cdot 2$ & $24 \cdot 7$ & ND \\
\hline \multirow[t]{2}{*}{ Serovar B } & EB & 0.69 & 14.9 & 1.4 & $14 \cdot 1$ & 11.7 & 6.7 & $11 \cdot 7$ & 0.04 \\
\hline & RB & 0.60 & $40 \cdot 6$ & $35 \cdot 2$ & $38 \cdot 3$ & $58 \cdot 7$ & $32 \cdot 0$ & $41 \cdot 5$ & $4 \cdot 7$ \\
\hline \multirow[t]{2}{*}{ Serovar C } & EB & 0.27 & $4 \cdot 6$ & 5.9 & ND & $5 \cdot 4$ & 3.7 & $10 \cdot 4$ & 0.73 \\
\hline & RB & $0 \cdot 31$ & $24 \cdot 6$ & $23 \cdot 0$ & ND & 26.9 & $20 \cdot 1$ & $34 \cdot 7$ & $4 \cdot 1$ \\
\hline \multirow[t]{2}{*}{ Serovar D } & EB & 0.5 & ND & ND & ND & ND & $10 \cdot 1$ & $16 \cdot 6$ & ND \\
\hline & RB & 1.0 & ND & ND & ND & ND & $30 \cdot 2$ & $22 \cdot 9$ & ND \\
\hline \multirow[t]{2}{*}{ Serovar F } & EB & 0.1 & $13 \cdot 6$ & $5 \cdot 3$ & $10 \cdot 4$ & $14 \cdot 3$ & $14 \cdot 2$ & $25 \cdot 7$ & ND \\
\hline & $\mathbf{R B}$ & 0.59 & $25 \cdot 1$ & $13 \cdot 5$ & $37 \cdot 2$ & $34 \cdot 1$ & $35 \cdot 1$ & $45 \cdot 1$ & ND \\
\hline \multirow[t]{2}{*}{ Serovar G } & EB & ND & ND & ND & ND & $\mathrm{ND}$ & $11 \cdot 1$ & $12 \cdot 2$ & ND \\
\hline & $\mathbf{R B}$ & ND & ND & ND & ND & ND & $33 \cdot 8$ & ND & ND \\
\hline \multirow[t]{2}{*}{ Serovar Ll } & EB & 0.14 & 15.9 & 5.5 & $12 \cdot 0$ & 17.9 & $12 \cdot 1$ & $19 \cdot 3$ & 0.18 \\
\hline & RB & 0 & $51 \cdot 3$ & $41 \cdot 0$ & $89 \cdot 9$ & $48 \cdot 2$ & $60 \cdot 2$ & $49 \cdot 1$ & $3 \cdot 4$ \\
\hline \multirow[t]{2}{*}{ C. psittaci } & EB & 0.36 & $0 \cdot 6$ & 0.4 & $1 \cdot 3$ & 0.5 & 0.2 & 0.3 & ND \\
\hline & RB & $2 \cdot 2$ & $3 \cdot 1$ & 1.5 & $3 \cdot 4$ & $6 \cdot 2$ & 1.8 & 0.9 & ND \\
\hline
\end{tabular}

ND, Not determined.

* Pre-immune serum from rabbit As6. Essentially similar results were obtained for each serovar on at least two separatẹ occasions.

the VS III peptides recognized by As5 may be molecular mimics (mimotopes) of natural epitopes present elsewhere in the protein and recognized by low stringency antibodies. Antibodies reactive with peptides spanning the variable loop domains of MOMP were predominant.

\section{Discussion}

The immunopathological side effects of immunization with whole-cell chlamydial preparations has emphasized the need for a subunit vaccine. One difficulty with this approach, is the large-scale production of distinct, purified antigens from this fastidious intracellular bacterium. For MOMP, one approach has been the identification of continuous $B$ cell epitopes using either hgt11-expressing fragments or synthetic peptides in conjunction with mAbs of known specificity (Baehr $e t$ al., 1988; Conlan et al., 1988; Stephens et al., 1988). This rationale may lead to a synthetic peptide vaccine. Indeed, antibodies raised to synthetic peptide homologues of MOMP, recognize the native structure on EB (Conlan et al., 1989b). However, the presentation of suitably adjuvanted synthetic peptides to the immune system and the probable requirements for additional $T$ cell epitopes as well as carrier molecules remains problematic, especially as regards human usage (Shinnick et al., 1983). In these respects, larger recombinant proteins may be particularly useful in providing their own T-helper epitopes, thus making them inherently more immunogenic.

The ideal chlamydial vaccine should protect against infection with a wide spectrum of serovars. To date, neutralization studies have shown that antibodies reactive with serovar- and subspecies-specific epitopes exposed at the surface of the EB prevented chlamydial infectivity in vivo (Zhang et al., 1987) and in vitro (Caldwell \& Perry, 1982; Lucero \& Kuo, 1985). By contrast, antibodies directed against a hidden speciesspecific epitope did not abrogate infectivity (Zhang et al., 1987). Whether protection is a result simply of antibody binding to the MOMP surface is not known. The finding that antibody to the surface-accessible, genus-specific LPS failed to neutralize chlamydial infectivity (Zhang et al., 1987) might suggest that binding alone is insufficient for effective neutralization. In in vitro studies, MOMP-specific neutralizing antibodies did not prevent the attachment and uptake of EB, but did halt their differentiation to replicating RB (Caldwell \& Perry, 1982). This differentiation has been shown to be dependent upon relaxation of the sulphydryl cross-linkages in EB MOMP (Bavoil et al., 1984; Hatch et al., 1984). Neutralizing antibodies might also function by cross-linking the MOMP porin structure, thus preventing differentiation events. If this is so then antibodies solely directed to linear epitopes may not be protective. Although type-, subspecies- and speciesspecific MOMP domains consisting of short segments of 

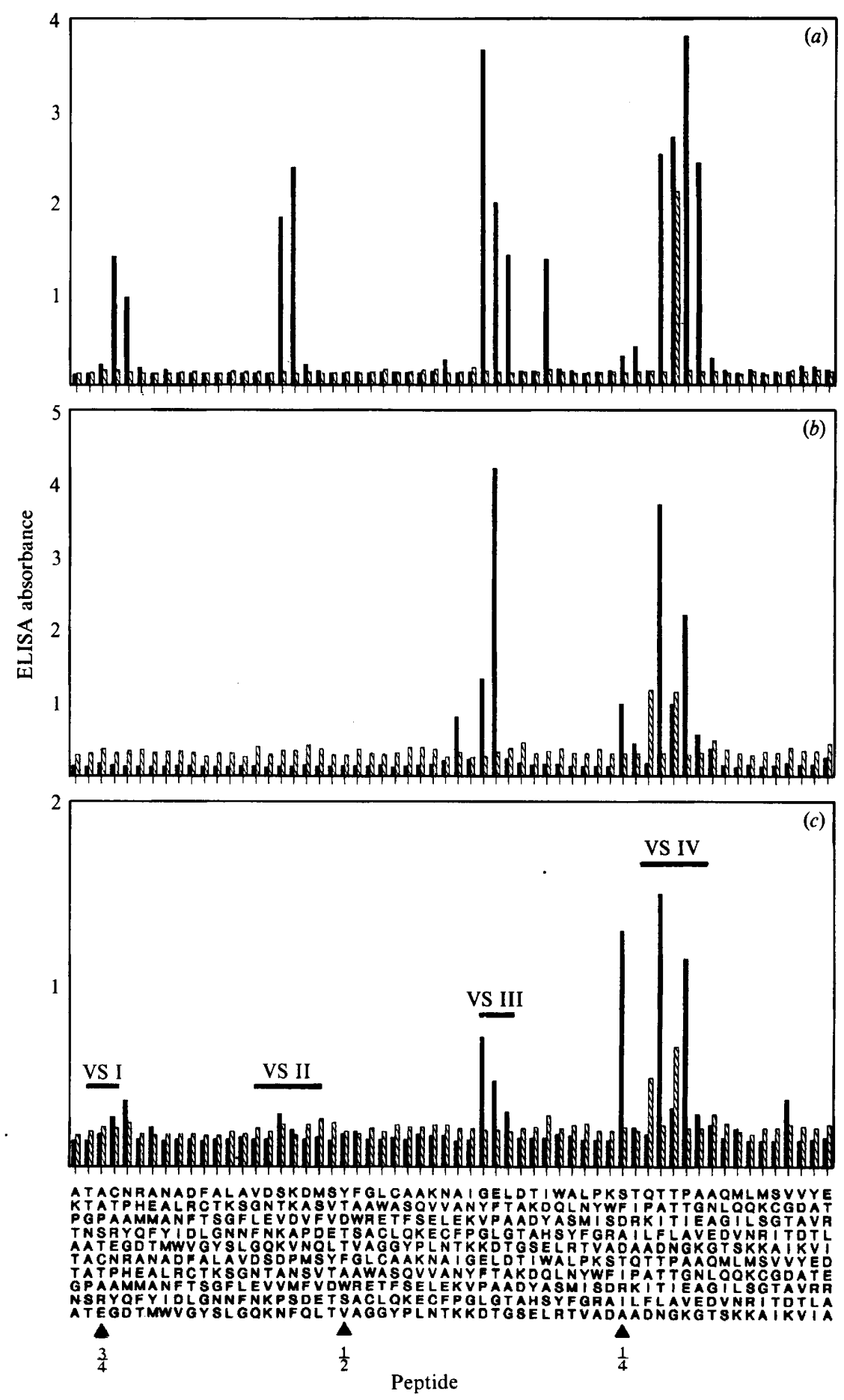

Fig. 4. Reactivity of rMOMP antisera with solid-phase synthetic peptides. As1, 3 and 5 were prepared using Freund's adjuvant. As2, 4

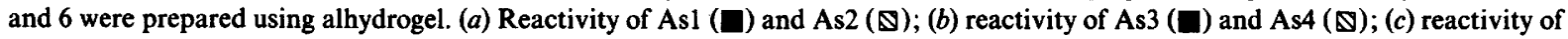
As5 $(\square)$ and As6 $(\mathbb{\Xi})$. The origins of $\frac{3}{4}, \frac{1}{2}$ - and $\frac{1}{4}$ MOMP translation are indicated. VS, variable segment.

continuous amino acid sequence have been identified, we have recently obtained evidence that more than one of these loop regions may be spanned by a single $\mathrm{mAb}$ paratope (Conlan et al., 1990). Thus, it is likely that these linear antigenic segments interact to form the paratope contact regions of a discontinuous epitope.

The rMOMP fragments used in the present study were expressed as insoluble inclusions located in the cyto- 
plasm (Pickett et al., 1988b). This enabled substantial purification by the simple expedient of spheroplasting followed by lysis and centrifugation. Although immunochemical assays demonstrated the presence of contaminating $E$. coli antigens in the recombinant preparations, these appeared to be minor components, possibly associated with host-cell ribosomes occluded within the rMOMP inclusions. Control experiments showed that antibodies raised against contaminating antigenic material were not responsible for the specific reactivity with chlamydiae of antisera to rMOMP. The multiple bands observed on Western-blotting the rMOMP preparations against a MOMP-specific mAb indicated the presence of MOMP aggregates and processing fragments. These may also occur in chlamydiae themselves, which might explain the multiple bands of $C$. trachomatis antigens reactive with high titre antisera to the rMOMP.

Immunization with all three rMOMP fragments produced Chlamydia genus-reactive antibodies by ELISA and Western blotting. However, in immunogold assays, where intact chlamydiae were labelled in suspension, only $C$. trachomatis species-reactive antibodies were detected. This emphasizes the importance of using native bacteria whose topography is unaltered by binding to a solid phase when searching for immunoaccessible surface epitopes. Whether those antibodies which reacted at the EB surface also possessed useful biological functions remains to be determined. It was encouraging, however, that even immunization with the smallest MOMP fragment produced antibodies to known neutralizing epitopes in VS IV, as this recombinant appeared to be the easiest to purify as assessed by SDS-PAGE and Western blots. Moreover, adsorption of recombinant fragments to alhydrogel, a humanapproved adjuvant, evoked antibodies to exposed epitopes of MOMP.

Epitope mapping studies with solid-phase peptides were employed in an attempt to explain the serological specificity of recombinant MOMP antisera in molecular terms. The antisera reacted almost exclusively with peptides spanning the variable segments of MOMP, as is the case for neutralizing MOMP mAbs produced by immunization with whole chlamydiae (Zhang et al., 1987). This was surprising given the harsh denaturing conditions to which the MOMP fragments were subjected prior to immunization, but it offers the possibility that rMOMP might be useful for vaccine development. It is possible that MOMP fragments refolded into native conformation following isolation and solubilization. Alternatively, the variable segments may contain epitopes which are immunodominant because they occur in mobile loop regions or because of the functional juxtaposition of strong T-helper epitopes. An examination of the peptides reacting with MOMP antisera did not provide a ready explanation for the serological reactivity observed in immunochemical assays with whole or denatured organisms. Thus, none of the antisera bound to peptides containing genus-specific sequences, despite their reactivity with denatured $C$. psittaci antigens on Western blot and in ELISA. All of the antisera reacted with peptides from VS IV, even though the species-specific peptide LNPTIAG located in this region is only poorly exposed, if at all, on the EB surface of most serovars (Conlan et al., 1989a; Kuo \& Chi, 1987; Zhang et al., 1987). Hence, this reactivity is unlikely to account for the observed species-specific labelling of intact chlamydiae. However, VS IV appears to be immunologically complex, as two surface-exposed, subspecies-specific epitopes flank each side of the speciesspecific region (Conlan et al., 1988, 1989b).

MOMP is currently the main candidate for the development of a chlamydial vaccine. Monospecific polyclonal antibodies to MOMP, with their high binding affinity, may also be useful for the development of diagnostic tests for chlamydial antigen. The observation reported here that rMOMP preparations in conjunction with human-acceptable alhydrogel adjuvant generate high-titre antibodies, of similar epitope-specificity to mAbs produced by immunization with whole chlamydiae (Conlan et al., 1988), is therefore of major interest. Collaborative studies in progress also indicate that these same rMOMP preparations can be presented, via dendritic cells, to stimulate primary and secondary $T$ cell responses (Dr A. Stagg, personal communication). The ability of rMOMP fragments to stimulate protective immunity in vivo is currently being investigated using a mouse model of human $C$. trachomatis infection.

This work was supported by grants from the Edna McConnell Clark Foundation and the Human Reproduction Programme of the World Health Organization.

\section{References}

Baehr, W., Zhang, Y.-X., Joseph, T., Su, H., Nano, F. E., EveretT, D. E. \& CAlDWell, H. D. (1988). Mapping antigenic domains expressed by Chlamydia trachomatis major outer membrane protein (MOMP) genes. Proceedings of the National Academy of Sciences of the United States of America 85, 4000-4004.

Batteiger, B. E., Newhall, W. J., Terho, V. P., Wilde, C. E. \& JONES, R. B. (1986). Antigenic analysis of the major outer membrane protein of Chlamydia trachomatis with murine monoclonal antibodies. Infection and Immunity 53, 530-533.

Bavoil, P., Ohlin, A. \& Schachter, J. (1984). Role of disulfide bonding in outer membrane structure and permeability in Chlamydia trachomatis. Infection and Immunity 44, 479-485.

Caldwell, H. D. \& PerRy, L. (1982). Neutralization of Chlamydia trachomatis infectivity with antibodies to the major outer membrane protein. Infection and Immunity 38, 745-754.

Caldwell, H. D., Kromhout, J. \& Schachter, J. (1981). Purification and partial characterization of the major outer membrane protein of Chlamydia trachomatis. Infection and Immunity 31, 1161-1176. 
Collett, B. A., Newhall, W. J., Jersild, V. R. A. \& Jones, R. B. (1989). Detection of surface-exposed epitopes on Chlamydia trachomatis by immune electron microscopy. Journal of General Microbiology 135, 85-94.

Conlan, J. W., Clarke, I. N. \& Ward, M. E. (1988). Epitope mapping with solid phase peptides: identification of type-, subspecies-, species-, and genus-reactive antibody binding domains on the major outer membrane protein of Chlamydia trachomatis. Molecular Microbiology 2, 673-679.

Conlan, J. W., Kajbaf, M., Clarke, I. N., Chantler, S. \& Ward, M. E. $(1989 a)$. The major outer membrane protein of Chlamydia trachomatis: critical binding site and conformation determine the specificity of antibody binding to viable chlamydiae. Molecular Microbiology 3, 311-318.

Conlan, J. W., Ferris, S., Clarke, I. N. \& Ward, M. E. (1989b). Surface-exposed epitopes on the major outer-membrane protein of Chlamydia trachomatis defined with peptide antisera. Journal of General Microbiology 135, 3219-3228.

Conlan, J. W., Persson, K., Newhall, W. J. \& Ward, M. E. (1990). Mapping of discontinuous epitopes on the major outer membrane protein of Chlamydia trachomatis using synthetic peptides and monoclonal antibodies. Proceedings of the 7th International Symposium on Human Chlamydial Infections (in the Press).

Geysen, H. M., Meloen, R. H. \& Barteling, S. J. (1984). Use of peptide synthesis to probe viral antigens for epitopes to a resolution of a single amino acid. Proceedings of the National Academy of Sciences of the United States of America 81, 3998-4002.

Hatch, T. P., Allan, I. \& Pearce, J. H. (1984). Structural and polypeptide differences between envelopes of infective and reproductive life cycle forms of Chlamydia spp. Journal of Bacteriology 157, 13-20.

Kuo, C. C. \& CHI, E. Y. (1987). Ultrastructural study of Chlamydia trachomatis surface antigens by immunogold staining with monoclonal antibodies. Infection and Immunity 55, 1324-1328.

LUCERo, M. E. \& KUO, C. C. (1985). Neutralization of Chlamydia trachomatis cell culture infection by serovar-specific monoclonal antibodies. Infection and Immunity 50, 595-597.

OsBorn, M. J. \& Munson, R. (1974). Separation of the inner (cytoplasmic) and outer membranes of gram negative bacteria. Methods in Enzymology 31, 642-653.

Pickett, M. A., Ward, M. E. \& Clarke, I. N. (1987). Complete nucleotide sequence of the major outer membrane protein gene from Chlamydia trachomatis serovar L1. FEMS Microbiology Letters 42 , 185-190.

Pickett, M. A., Everson, J. S. \& Clarke, I. N. (1988a). Chlamydia psittaci ewe abortion agent: complete nucleotide sequence of the major outer membrane protein gene. FEMS Microbiology Letters 55, 229-234.
Pickēt, M. A., Ward, M. E. \& Clarke, I. N. (1988b). High-level expression and epitope localisation of the major outer membrane protein from Chlamydia trachomatis serovar L1. Molecular Microbiology 2, 681-685.

RoBerTSON, J. N. \& WARD, M. E. (1988). Gonococcal and chlamydial infection in infertility and ectopic pregnancy. Contemporary Reviews in Obstetrics and Gynaecology 1, 60-66.

Robertson, J. N., Ward, M. E., Conway, D. \& CaUl, E. O. (1987). Chlamydial and gonococcal antibodies in sera of infertile women with tubal obstruction. Journal of Clinical Pathology 40, 377-383.

Salari, S. H. \& Ward, M. E. (1981). Polypeptide composition of Chlamydia trachomatis. Journal of General Microbiology 123, 197-207.

SCHACHTER, J. \& Dawson, C. (1978). Chlamydial Infections. Littleton, Massachusetts: PSG Publishing.

SHINNICK, T. M., SUTCLIFfe, J. G., GREEN, N. \& LEHNER, R. A. (1983). Synthetic peptide immunogens as vaccines. Annual Review of Microbiology 37, 425-446.

StePhens, R. S., SANChez-Pescador, R., WaGar, E. A., InUoye, C. \& URDEA, M. S. (1987). Diversity of Chlamydia trachomatis major outer membrane protein genes. Journal of Bacteriology 169, 3879-3885.

Stephens, R. S., WaGaR, E. A. \& SchOOLNIK, G. K. (1988). High resolution mapping of serovar-specific and common antigenic determinants of the major outer membrane protein of Chlamydia trachomatis. Journal of Experimental Medicine 167, 817-831.

Terho, P., Matikainen, M. T., Arstila, P. \& Treharne, J. (1982). Monoclonal type-specific antibodies for Chlamydia trachomatis LGV strains. In Chlamydial Infections. Proceedings of the Fifth International Symposium on Human Chlamydial Infections, pp. 231-234. Edited by P. A. Mardh, K. K. Holmes, J. D. Oriel, P. Piot \& J. Schachter Amsterdam: Elsevier Biomedical Press.

WaNG, S. P. \& Grayston, J. T. (1982). Micro-immunofluorescence antibody responses in Chlaymdia trachomatis infection: a review. In Chlamydial Infections. Proceedings of the Fifth International Symposium on Human Chlamydial Infections, pp. 301-316. Edited by P. A Mardh, K. K. Holmes, J. D. Oriel, P. Piot \& J. Schachter. Amsterdam: Elsevier Biomedical Press.

Yuan, Y., Zhang, Y.-X., WatKins, N. G. \& Caldwell, H. D. (1989) Nucleotide and deduced amino acid sequences for the four variable domains of the major outer membrane proteins of the 15 Chlamydia trachomatis serovars. Infection and Immunity 57, 1040-1049.

Zhang, X.-Y., Stewart, S., Joseph, T., Taylor, H. R. \& CaldWell, H. D. (1987). Protective monoclonal antibodies recognize epitopes located on the major outer membrane protein of Chlamydia trachomatis. Journal of Immunology 138, 575-581. 